\title{
Time Machine for Past and Future Travel
}

\author{
Manu Mitra \\ Department of Electrical Engineering, Alumnus of University of Bridgeport, Bridgeport, United States. \\ mmitra@my.bridgeport.edu
}

\begin{abstract}
Time Machine is the journey between from certain points of time analogous to journey between various points in space by an object or a person. There has been significant development in Time Machine, but few depicts exact phenomenon and its effect.
\end{abstract}

This paper discusses about design of and structure for a Time Machine; in this paper time machine software and special relativity of Einstein is discussed that can lead past or future using coordinates. But one should know that that for any smallest change in past or future could change entire present.

Index Terms : Time Machine, Design, Construction

\section{Introduction}

It is undefined that if time travel to the past is physically possible. But time travel in in future is the sense of perception of time. It is broadly observed phenomenon and well comprehended within the framework of special relativity and general relativity. Nevertheless, movement of one body advance or delay more than a few milliseconds compared to another body is being researched with given challenge of current technology.

As for backward time travel is possible to find solutions in general relativity that allow for it for instance rotating black hole. However, moving a body in spacetime to a random point has very limited information in theoretical physics and typically connected with quantum mechanics or other means such as worm hole. [1]

\section{WORKING PRINCIPLE OF TIME MACHINE}

In the above Fig.1. illustrates the working principle of Time Machine. In this picture a nonlinear crystal method is used to create two entangled photons, for one of it is being sent through the Einstein-Bose condensate which arrives later than double slit.

As per the illustration it will lead to earlier arriving photon (2) to not create an interference pattern in case it has been collapsed in future. One can simply imagine to do this on a hundred or more photons. If only one hundred photons would appear on the screen where it should not appear, hence there would be breaks normally because of the interference, therefore we would know that 1 of 100 delayed photons at least was detected.

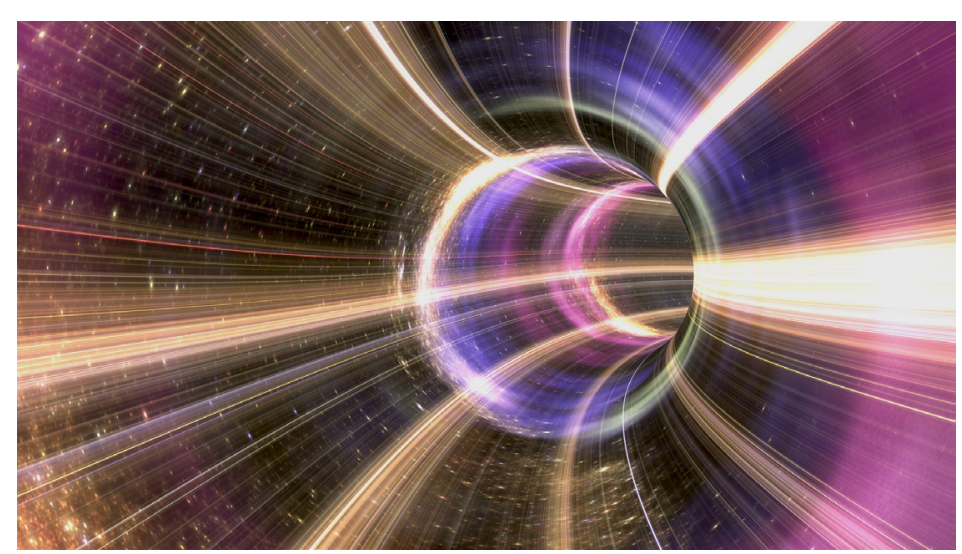

Figure 1: Illustrates the wormhole in the space [2] 
One could build a second device and send the information when it is received another time back, moving further back in time, then a third and so on or one could just use the same machine and do it again, looping the output into the input. There would be only restricted to the point where one created the device and wouldn't be able to send information back any further.

It would mean that you cannot possibly act on the information you receive like if you were a Zombie or one would try; then universe would make sure that something really atrocious happened to you, and the sender in the future is actually someone else or other unbelievable, impossible sounding explanations. [3]

\section{SOFTWARE OF TIME MACHINE}

Artificial Intelligence (AI) can fabricate future pathways hierarchically and reconstruct the future pathways, AI also

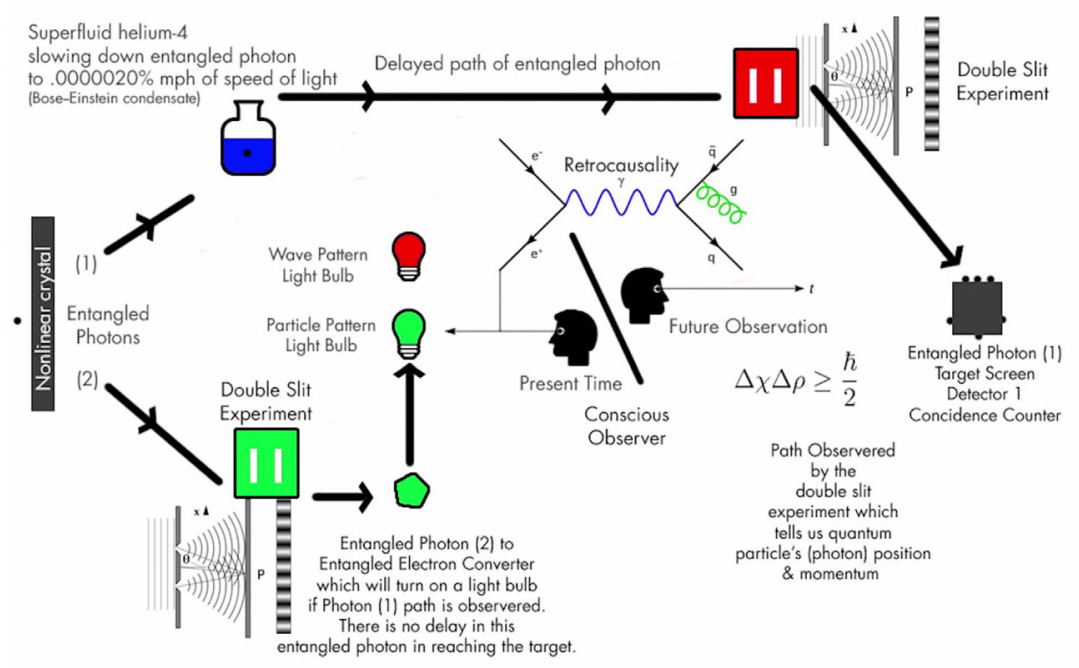

Figure 2: IIllustrates the communication in the 4th dimension to the entangled Photon (2) to either act like a wave or a particle based on if observe the particles position and momentum in the future. This technology is based on m-theory [3]
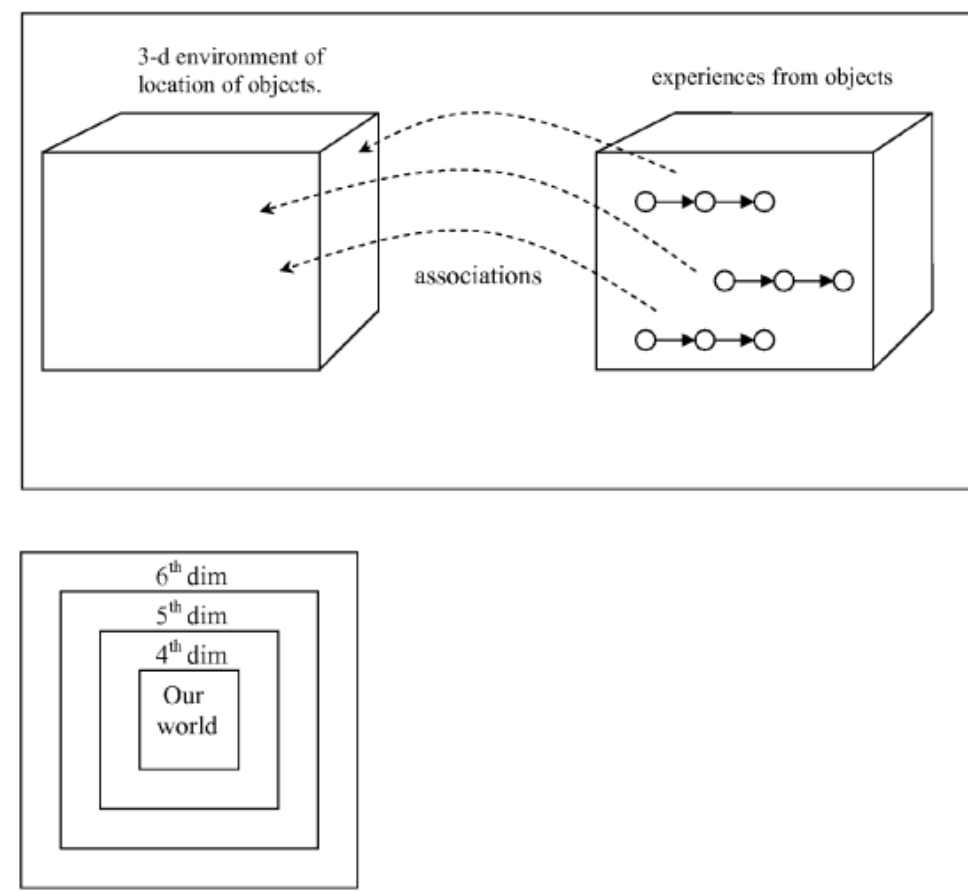

Figure 3: Illustrates prediction of universal pathways based on a human beings actions [4] 
matches each future pathway to the pathways in the time machine. Time Machine is used to give future pathways a more accurate and realistic prediction. Since Time Machine is collection of pathways from multiple robots and hence it's accurate and detailed. [4]

Figure 4 illustrates software is in the loop to find the best match for the coordinates assigned in the time machine.

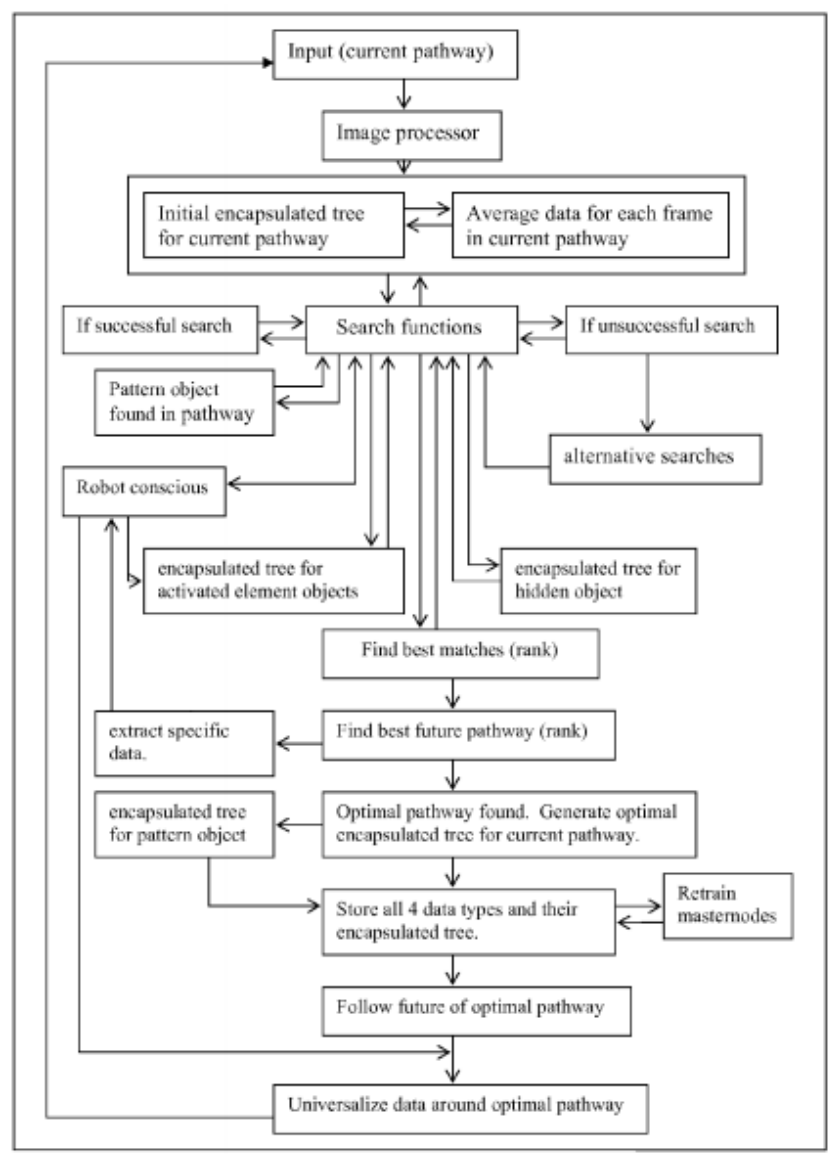

Figure 4: Illustrates prediction paths for Time Travel [4]

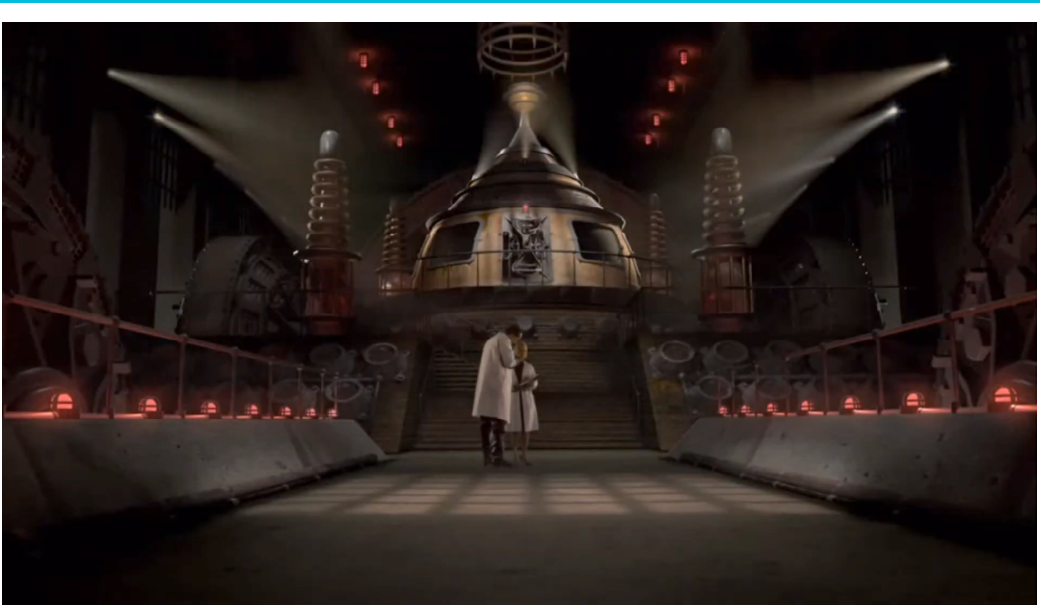

Figure 5: Design Concept of a Time Machine 


\section{DESIGN ARCHITECTURE OF A TIME MACHINE}

Explanation of illustrations - Figure 4 shows sample of time machine with dome like structure and two tesla coils powering the machine with high voltage. Second machine is not assumed for the ease of understanding. Figure 5 shows dome structure of time machine with two unpowered tesla coils. Figure 6 shows powering of time machine with tesla coils and people inside the machine are teleported to past or future as per the coordinates assigned in the time machine (not shown). Figure 7 shows Internal design for three seats for three people including time machine engineer. Figure 8 shows time machine when it is switched off and unpowered. It is shown after the teleportation of users inside the time machine. Figure 9 shows when users coming back to present time.

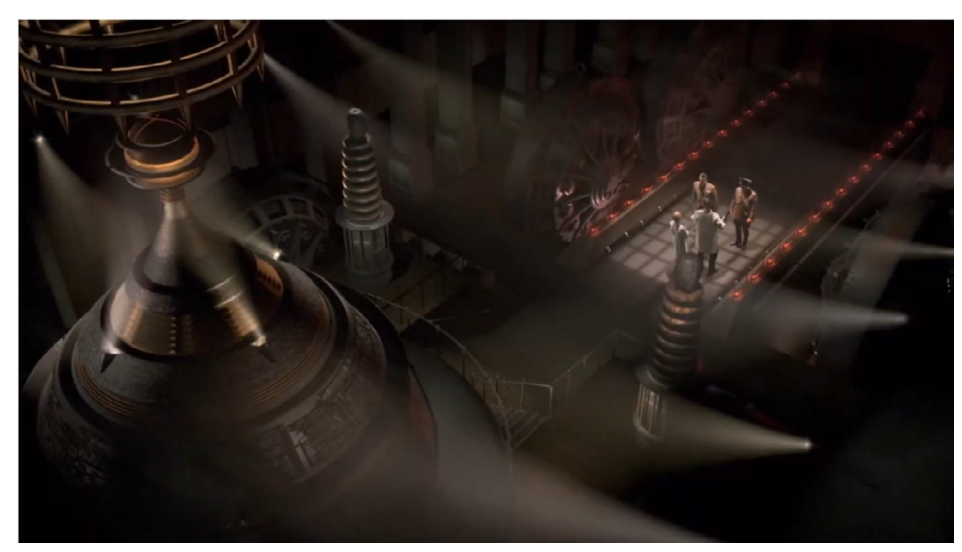

Figure 6: Design Concept of a Time Machine Dome view

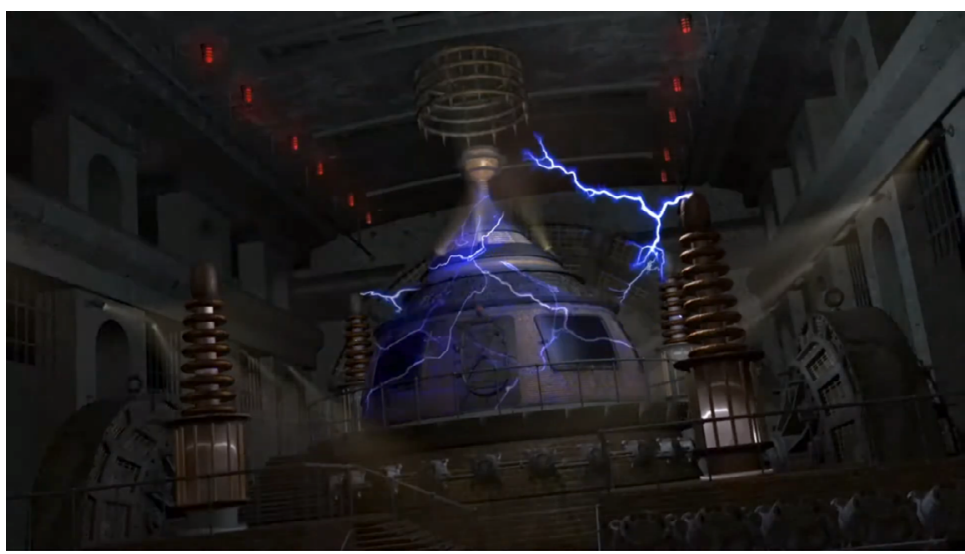

Figure 7: Time Machine Powered by Tesla Coils to give energy to travel

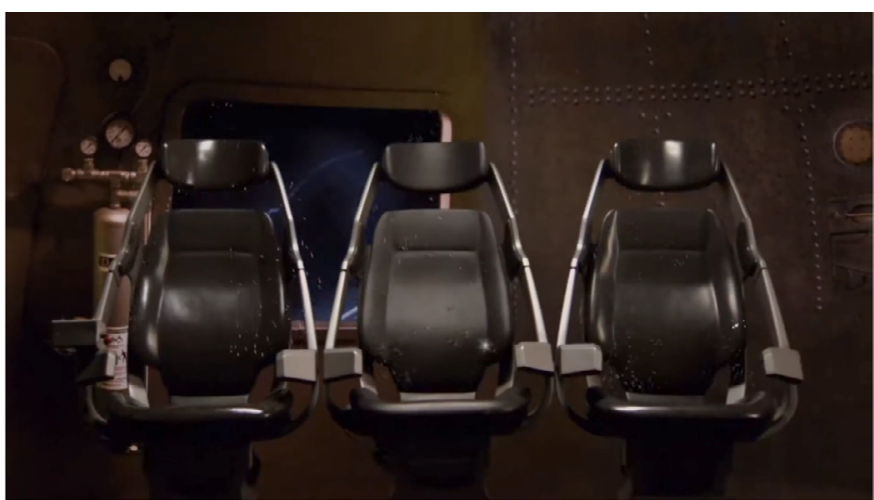

Figure 8: Internal design of Time Machine for three people 


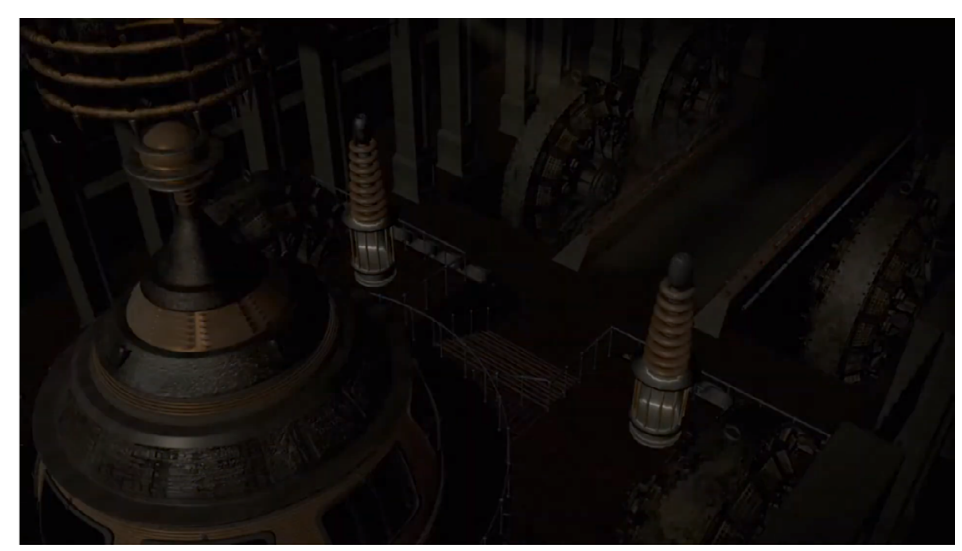

Figure 9: Time Machine when it is switched off

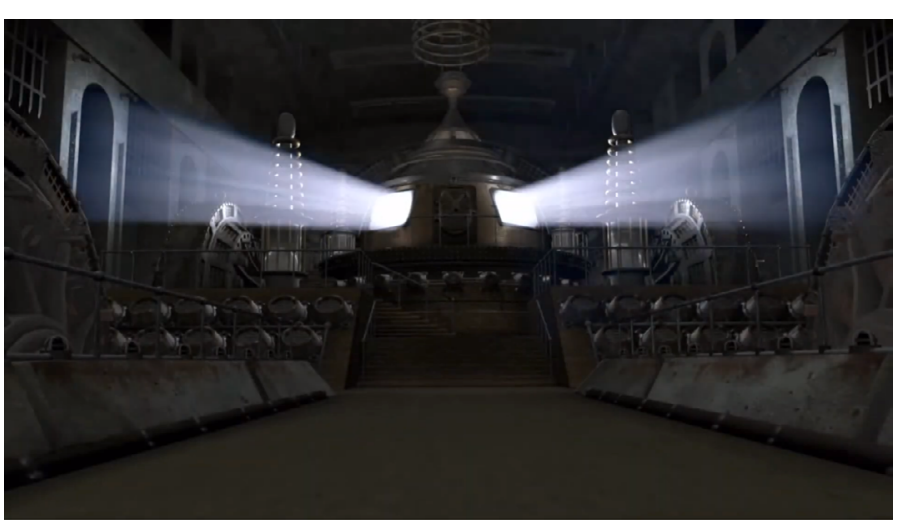

Figure 10: Time Machine when users comes back to present

Note: Time Machine may only be used to view, any alteration in past or future affects present very drastically.

\section{SPECIAL RELATIVITY IN TIME TRAVELLING}

As per Maxwell's equations of electromagnetics; speed of a light wave is independent of reference frame, that is observed in. It is plainly violation of Galilean transformation rules.

As per Galilean laws if an observer in frame (S'), moving with velocity (v), sends out a light signal, with velocity (c), in the direction of travel then the velocity of the light wave is measured by a stationary observer in the frame $\mathrm{S}$,

$$
\begin{gathered}
x^{\prime}=\gamma(x-v t) \\
y^{\prime}=y \\
z^{\prime}=z \\
t^{\prime}=\gamma\left(t-\frac{v}{c^{2}} x\right)
\end{gathered}
$$

Where $\gamma$ is defined as:

$$
\gamma \equiv \frac{1}{\sqrt{1-\frac{v^{2}}{c^{2}}}}
$$

Einstein recognized physical significance of this transformation and thus formulated special theory. The transformations now show that time is not the absolute dimension we thought it was. Later discussion of time travel opportunities are presented in this theory. However, it is important to note that in the Lorentz transformation there looks to be mixture of space and time coordinates. It was at this point that people began to realize that space and time were not the separate entities that they appear to be. They are bound together in some or other way in a 4-dimensional spacetime continuum.

Before moving to time in General Relativity it will be useful for me to digress into some mathematical formalism that will be useful in our later discussions of spacetime warps. In relativity it is useful to talk about metrics that describes 
the geometry of spacetime. Often, we find a metric that can describe behavior of spacetime in a specific region. By metric, it is an equation that is written by computing the distance element of a spacetime in terms of the coordinate system. For example, in a 2-dimensional flat space with Cartesian coordinates, $\mathrm{x}$ and $\mathrm{y}$, the distance, Delta $\mathrm{s}$, can be found by forming a right triangle and applying the Pythagorean theorem

$$
s^{2}=\Delta x^{2}+\Delta y^{2}
$$

We often write this in as an infinitesimal distance element, ds. In three-dimensional space we have

$$
d s^{2}=d x^{2}+d y^{2}+d z^{2}
$$

After a while, Einstein published his first paper on special relativity, Rudolph Minkowski identified that the Lorentz transformation described a 4-dimensional spacetime with the metric equation

$$
d s^{2}=c^{2} d t^{2}-d x^{2}-d y^{2}-d z^{2}
$$

In this measured cadent there are two different times. First is the coordinate time, the time measured by two stationary observers to the coordinate system. Second one is the proper time, the time measured cadent by a lone observer whom measures their own motion to be zero. The proper time is found by dividing the distance in the spacetime by a velocity, the speed of light.

$$
\text { ProperTime }=d \tau=\frac{d s}{c}
$$

The proper time and the coordinate time are related by the metric equation.

$$
d t=\gamma d \tau, \gamma \equiv \frac{1}{\sqrt{1-\frac{v^{2}}{c^{2}}}}
$$

This relation will help us travel in time [5]

\section{TIME AS PHYSICAL DIMENSION}

Based on the illustration of Figure 9, if it takes one second for cross sections to travel along each extrusion from one place to next, then all rooms in the above picture to the future of image o by the number of seconds shown in black in the room Number 2 is the second ahead of room 0 , room 9 is two seconds ahead of room 0 and room 8 is 4 seconds ahead of room 0 and so on.

To travel into the future, one may want to consider to move diagonal of the compartment in the direction of increasing blue, green and brown time (rightward, upward and inward) that is, along the diagonal dashed violet line. [6]

\section{CONCLUSION}

1. Time Travel design example is shown and is powered by Nikola Tesla’s Tesla Coils.

2. Time as physical dimension is illustrated based on Einstein's special relativity

3. Based on the Einstein's Special relativity Time travel is shown.

\section{References}

1. Wikipedia. (2001, August 9). Time travel. In Wikipedia, the free encyclopedia. https://en.wikipedia.org/wiki/Time_travel

2. Krauss, L. M. (2017, May 10). What Einstein and Bill Gates teach us about time travel. NBC News. https://www.nbcnews.com/storyline/the-bigquestions/what-einstein-bill-gates-teach-us-about-time-travel-n757291

3. Stack Exchange. (2015, April 23). Is this a working time machine? Physics Stack Exchange. https://physics.stackexchange.com/questions/177940/isthis-a-working-time-machine?noredirect=1\&lq=1

4. Kwok, M. (2008, November 13). United States patent application: 0080281766. USPTO. https://appft1.uspto.gov/netacgi/nph-Pars er? Sect $1=$ PTO $1 \&$ Sect $2=$ HITOFF $\& d=P G 01 \& \mathrm{p}=1 \& \mathrm{u}=/$ netahtml $/$ $\mathrm{PTO} / \mathrm{srchnum} . \mathrm{html} \& \mathrm{r}=1 \& \mathrm{f}=\mathrm{G} \& \mathrm{l}=50 \& \mathrm{~s} 1=20080281766$. PGNR.\&OS=DN/20080281766

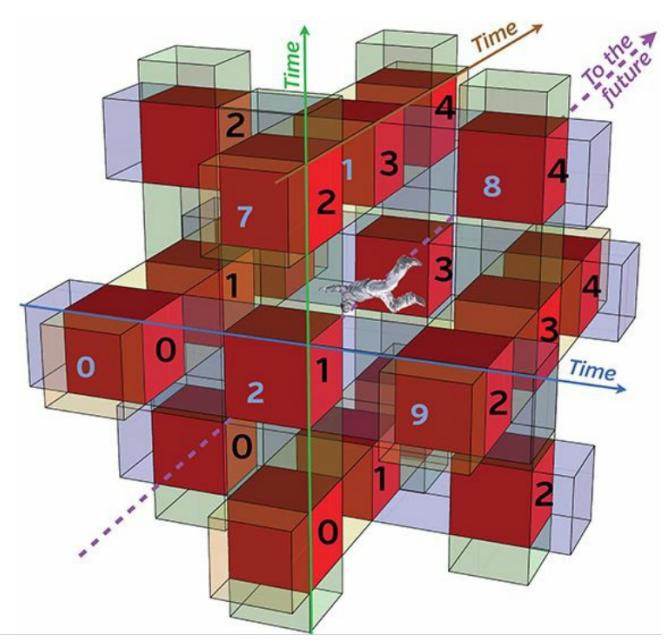

Figure 11: This picture illustrates time as physical dimension and how it move in future with Einstein's special relativity [6] 
5. Méndez, B. J. (2020, August 1). Time travel. Berkely. https://cse.ssl. berkeley.edu/bmendez/html/time.html
6. Zamandayolculuk. (2020, August 1). Interstellar- Voyage. Zamanda Yolculuk - Giriş Sayfası. https://www.zamandayolculuk.com/html-2/ interstellar-voyage.htm

Citation: Manu Mitra "Time Machine for Past and Future Travel". American Research Journal of Electronics and Communication Engineering, vol 2, no. 1, 2020, pp. 1-7.

Copyright (C2020 Manu Mitra. This is an open access article distributed under the Creative Commons Attribution License, which permits unrestricted use, distribution, and reproduction in any medium, provided the original work is properly cited. 